\title{
A distal effect of microsomal triglyceride transfer protein deficiency on the lysosomal recycling of CD1d
}

\author{
Yuval Sagiv, ${ }^{1}$ Li Bai, ${ }^{1}$ Datsen G. Wei, ${ }^{1}$ Reuven Agami, ${ }^{3}$ Paul B. Savage, ${ }^{4}$ \\ Luc Teyton, ${ }^{5}$ and Albert Bendelac ${ }^{1,2}$
}

'Committee on Immunology and ${ }^{2}$ Howard Hughes Medical Institute, University of Chicago, Chicago, IL 60637

${ }^{3}$ Division of Tumor Biology, The Netherlands Cancer Institute, 1066 CX Amsterdam, Netherlands

${ }^{4}$ Department of Chemistry, Brigham Young University, Provo UT 84602

${ }^{5}$ Department of Immunology, The Scripps Research Institute, La Jolla, CA 92037

Microsomal triglyceride transfer protein (MTP) is an endoplasmic reticulum (ER)-resident lipid transfer protein involved in the biosynthesis and lipid loading of apolipoprotein B. MTP was recently suggested to directly regulate the biosynthesis of the MHC I-like, lipid antigen presenting molecule CD1d, based on coprecipitation experiments and lipid loading assays. However, we found that the major impact of MTP deficiency occurred distal to the ER and Golgi compartments. Thus, although the rates of CD1d biosynthesis, glycosylation maturation, and internalization from the cell surface were preserved, the late but essential stage of recycling from lysosome to plasma membrane was profoundly impaired. Likewise, functional experiments indicated defects of CD1d-mediated lipid presentation in the lysosome but not in the secretory pathway. These intriguing findings suggest a novel, unexpected role of MTP at a late stage of CD1d trafficking in the lysosomal compartment.

CORRESPONDENCE

Albert Bendelac:

abendela@bsd.uchicago.edu

Abbreviations used: GSL, glycosphingolipid; HEL, hen egg lyzozyme; HRP, horse radish peroxidase; KD, knock down; MTP, microsomal triglyceride transfer protein; RBL, rat basophilic leukemia; siRNA, small interfering RNA.
The CD1 family of glycoproteins is composed of conserved MHC-like, $\beta 2$-microglobulinassociated glycoproteins that specialize in the capture of self and microbial lipid antigens for presentation to $\mathrm{T}$ cells $(1,2)$. Recent studies have revealed that CD1-mediated antigen presentation depended on a set of proteins involved in general lipid metabolism. Thus, lysosomal saposins, which promote the enzymatic degradation of glycosphingolipids (GSLs), also performed essential lipid exchange reactions between membranes and CD1 proteins (3-5). CD1e, a lysosomal member of the human CD1 family, enhanced lipid processing by degrading enzymes (6). Serum VLDL and the surface LDL receptor directly contributed to the transport and uptake of exogenous lipids (7). Disruption of NPC1, a transmembrane protein present in late endosomal membranes, interrupted GSL trafficking from late endosome to lysosome and impaired CD1-mediated antigen presentation and NKT cell development (8).

Several lines of evidence also suggest a role for microsomal triglyceride transfer protein (MTP), an ER-resident protein that functions as a lipid transfer protein and is essential for the loading of apolipoprotein B (apoB) with cholesterol, triacylglycerol, and phospholipids (9). MTP was originally characterized as a heterodimer of protein disulfide isomerase and a 97-kD subunit in the ER of hepatocytes and enterocytes (10-12), but recent studies have demonstrated weak expression in hemopoietic cells as well, including $T$ cells and dendritic cells (13). Because MTP coprecipitated with CD1d and could transfer lipids onto plate-bound CD1d in a cell-free assay, it was proposed that MTP might assist in loading lipids onto CD1 molecules during biosynthesis in the ER in a manner similar to chaperone-assisted loading of peptides onto nascent MHC class I molecules $(13,14)$. In the absence of MTP, misfolded CD1d molecules would be retained in the ER, explaining the reduction of surface CD1d and the impaired antigen presentation observed in MTP-deficient cells. Consistent with this hypothesis, MTP ablation after injection of double-stranded RNA (dsRNA [polyI:C]) in $m t t p^{\mathrm{f} / \mathrm{fl}} \mathrm{Mx} 1-\mathrm{Cre}$ mice afforded resistance to diseases mediated by CD1d-restricted NKT cells such as $\alpha$ GalCer-induced hepatitis and oxazolone-induced colitis (14).

Here, we have examined the presentation of lipid antigens and studied the dynamics of 
the cellular trafficking of CD1d and lipids in cells lacking MTP. Severe defects in lipid antigen presentation were observed, but, surprisingly, they appeared to be selective for lipid antigens requiring lysosomal processing or loading rather than those acquired in the secretory pathway. In addition, cell biological assays revealed that MTP deficiency selectively impaired CD1d trafficking between the lysosome and the plasma membrane, far from the proposed site of action during biosynthesis in the ER. Consistent with these findings, the development of $\mathrm{V} \alpha 14-\mathrm{J} \alpha 18 \mathrm{NKT}$ cells, which requires lysosomal loading of natural ligands, was partially impaired in radiation chimeras reconstituted with MTP-deficient bone marrow cells. These intriguing findings converge to define a novel MTP-regulated mechanism that controls an essential step in the presentation of many lipid antigens, the recycling of CD1d from the lysosome to the plasma membrane.

\section{RESULTS}

\section{MTP ablation impairs Vo14 NKT cell development}

Because expression of CD1d by cortical thymocytes is essential for the development of V $\alpha 14$ NKT cell, we crossed $m t t p^{\mathrm{f} / \mathrm{fl}}$ mice to mice expressing the pLck-Cre transgene expressed in thymocytes. Genomic typing of WT and $m t t p^{\mathrm{A} / \mathrm{fl}}$ mice is shown in Fig. 1 A. Despite $~ 95 \%$ genetic ablation of the floxed mttp gene segment (Fig. $1 \mathrm{~B}$ ), the frequencies of V 114 NKT cells in thymus and spleen were not significantly diminished (Fig. 1 C). As an assay to probe for NKT ligand expression by thymocytes, we measured IL-2 release after exposure of NKT hybridomas to MTP-deficient thymocytes. Despite the absence of NKT cells' developmental defect, the response of the $\mathrm{V} \alpha 14$ hybridoma DN32.D3 was reduced, whereas, in contrast, the non-V $\alpha 14$ hybridoma TCB11 was
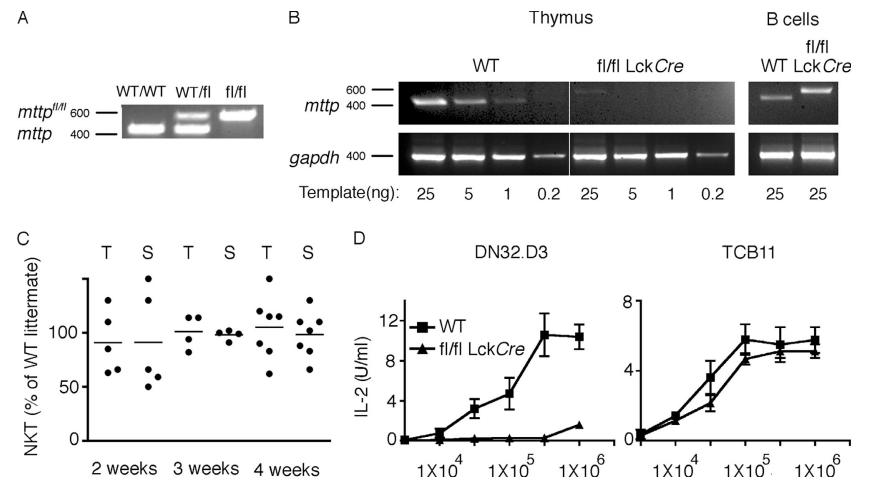

Figure 1. Impaired stimulation of $\mathrm{V} \alpha 14-\mathrm{J} \alpha 18$ NKT cells by $m t t p^{\mathrm{fl} / \mathrm{fl}}$ Lck-Cre thymocytes. (A) Genomic DNA typing of WT, mttp fl|fl, and heterozygous mice. (B) Semiquantitative PCR of genomic mttp and gapdh in thymocytes and sorted splenic B cells. (C) V $\alpha 14-J \alpha 18$ NKT cells in the thymus (T) and spleen (S) as determined by CD1d- $\alpha$ GalCer tetramer staining. Frequency of $V \alpha 14-J \alpha 18$ NKT cells are indicated as a percentage of mttp fl/fl Lck-Cre over WT littermates. (D) IL-2 response of the $\mathrm{V} \alpha 14-$ J 18 NKT hybridoma DN32.D3 and the non-V $\alpha 14$ hybridoma TCB11 stimulated by fresh thymocytes of WT and mttp ${ }^{\mathrm{fl} / \mathrm{fl}}$ Lck-Cre littermates as indicated. Mean \pm SD of two individual thymuses is shown; data are representative of two independent experiments. unaffected (Fig. 1 D). These hybridomas are widely used to probe for endogenous ligands acquired in the lysosomal versus the secretory pathway, respectively. Thus, DN32.D3 responds to iGb3 loaded onto CD1d by saposins in the lysosome, whereas TCB11 responds to an unidentified ligand loaded in the secretory pathway. Because the ablation of mttp was incomplete and low residual ligand expression could explain conserved NKT cell development in vivo, we crossed $m t t p^{\mathrm{f} / \mathrm{fl}}$ mice to mice expressing the Cre recombinase under control of the IFN-inducible $\mathrm{Mx} 1$ promoter. Bone marrow cells from $m t t p^{\mathrm{f} / \mathrm{fl}} \mathrm{Mx1}-\mathrm{Cre}$ (fl/fl-Mx1Cre) and WT (nonfloxed $m t t p)$ littermates treated with multiple injections of dsRNA (polyI:C) were used to reconstitute lethally irradiated CD $1 d^{-1-}$ hosts. This procedure achieved $99.6 \%$ deletion of mttp in the bone marrow (Fig. 2 A). In this system, V $\alpha 14$ NKT cells were modestly reduced by $50-60 \%$ both in the thymus and the spleen, whereas B cells and CD4 and CD8 T cells were preserved (Fig. 2, B and C). To test whether the

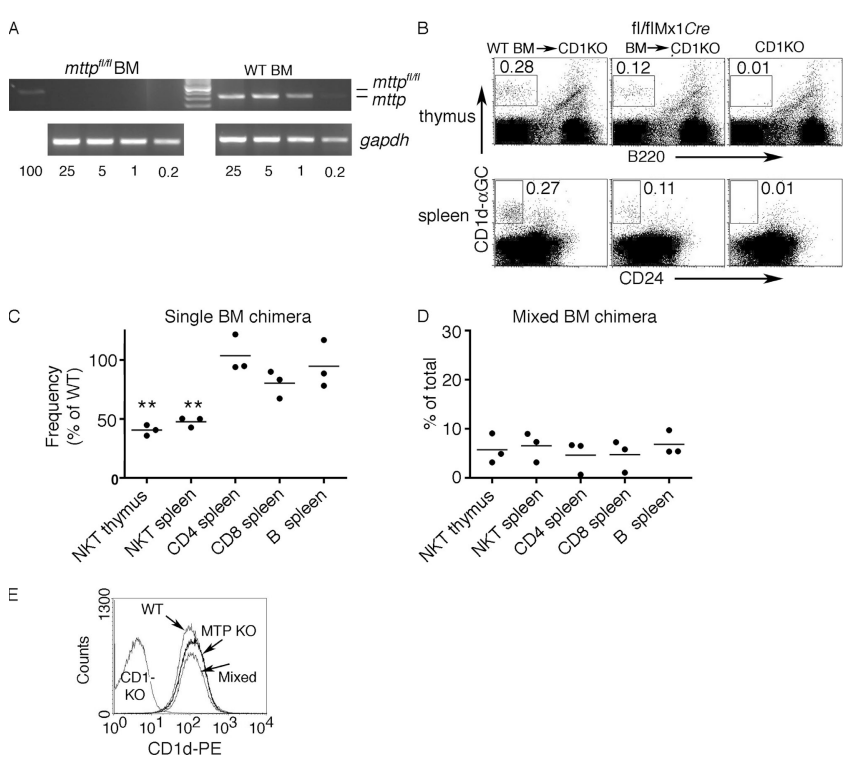

Figure 2. $m t t p^{\mathrm{fl} / \mathrm{fl}} \mathrm{Mx1}$-Cre bone-marrow reconstituted chimeras. Lethally irradiated $\mathrm{CD} 1 \mathrm{~d}^{-1-}$ mice were reconstituted with bone marrow (BM) cells from WT (nonfloxed $m t t p$ ) and $m t t p^{f / / f l} \mathrm{Mx} 1$-Cre littermates treated with multiple injections of dsRNA (polyl:C; see Materials and methods). (A) Semiquantitative PCR of DNA levels for mttp and gapdh in bone marrow from WT and $m t t p^{f / / f l} \mathrm{M} \times 1$-Cre littermates treated with dsRNA (polyl:C). (B) Percentage of $V \alpha 14-J \alpha 18$ NKT cells (gated as CD1d- $\alpha$ GalCer ${ }^{+}$B220 low for splenocytes and CD1d- $\alpha$ GalCer ${ }^{+}$CD24 low for thymocytes) in chimeras reconstituted with WT or $m t t p^{f / f l} \mathrm{Mx} 1-\mathrm{Cre}$ BM. A CD $1 d^{-1-}$ mouse is shown as control. (C) V $\alpha 14-J \alpha 18$ NKT cells, CD 4 and CD8 $T$ cells, and $B$ cell frequencies in chimeras reconstituted with $m t t p^{f / f l} \mathrm{Mx} 1-C r e$ bone marrow shown as a percentage of cell frequencies in chimeras reconstituted with WT BM. ${ }^{* *}, P<0.05$. (D) Mixed chimeras reconstituted with WT and MTP-deficient BM (1:1). Summary plot showing individual frequencies of MTP-deficient NKT, CD4, CD8, and $B$ cells as a percentage of the total. (E) CD1d surface expression in CD4+CD8 + double-positive thymocytes from chimeras reconstituted with WT, MTP-deficient, or mixed BM. Data are representative of three independent experiments. 
requirement of $\mathrm{MTP}$ was intrinsic to the developing NKT cells or to the CD1d-presenting cells, we generated mixed bone marrow chimeras by mixing bone marrow from Ly5.1 WT mice and from dsRNA (polyI:C)-injected fl/fl-Mx1Cre mice (Ly5.2). Although the overall hemopoietic reconstitution by MTP-deficient bone marrow cells was less efficient than WT, NKT cell development was unaffected relative to other lymphocyte compartments, including CD4 and CD8 $\mathrm{T}$ cells and B cells (Fig. 2 D). Considering the conserved level of CD1d expression (Fig. 2 E), these results are consistent with a selective defect in lipid ligand presentation by CD1d-expressing thymocytes as a mechanism for the decreased frequency of NKT cells.

\section{Selective defect in lysosomal-dependent lipid antigen presentation}

To investigate the functional and cell biological defects of CD1d-mediated lipid antigen presentation in cells lacking MTP, we used small interfering RNA (siRNA) to knock down (KD) the mttp mRNA in the rat basophilic leukemia (RBL) cell line transfected with CD1d. Different siRNA were designed to generate several stable clones expressing various levels of residual mRNA (Fig. 3 A). Clones 3-9 and 20-1 expressing $<20 \%$ residual MTP lost the ability to stimulate 2/2 Vo14 NKT hybridomas, DN32.D3 and N383c (Fig. 3 B). Clone $4-6$, with only an $\sim 50 \%$ reduction of MTP mRNA, exhibited only a modest defect in stimulation. Notably, stimulation of 3/3 non-V 14 NKT hybridomas, TCB11, TBA7, and 1C8DC1, remained intact (Fig. 3 B). These selective defects match the well-established dichotomy between V $\alpha 14$ and non-V $\alpha 14$ NKT hybridomas with respect to their recognition of endosomal and nonendosomal ligands, respectively. They do not seem consistent, however, with the model suggesting that MTP critically regulates CD1d biosynthesis in the ER. Surface staining of CD1d in MTP-deficient clones showed a twofold reduction compared with the parental clone. Although these differences may be accounted for by interclonal variations, it is notable that the intracellular levels of CD1d were conserved or increased compared with WT. Thus, the intracellular/surface ratio was increased three- to fourfold in MTP KD cells, suggesting intracellular retention (Fig. 3 C). Notably, sorted RBL-CD1d cells expressing low levels of surface CD1d stimulated DN32.D3 well above the MTP-deficient RBL-CD1d clones, which express an intermediate level of surface CD1d, whereas stimulation of TBA7 was not altered by the absence of MTP (Fig. 3 D). Thus, a lower level of surface CD1d expression is unlikely to explain the selective V $\alpha 14$ NKT cell stimulation defect.

Additional antigen presentation experiments were performed using a battery of well-defined synthetic GSL antigens (Fig. 4). The MTP KD clone 3-9 exhibited a partial defect in the presentation of $\alpha \mathrm{GC}$, which is known to depend on lysosomal loading, at least partially, for recognition by V $\alpha 14$ NKT cells. The two disaccharide GSLs, $\alpha 1,2 \alpha$ GalCer (PBS18) and $\alpha$ 1,4 $\alpha$ GalCer (PBS19), require lysosomal removal of the outer galactose before recognition by

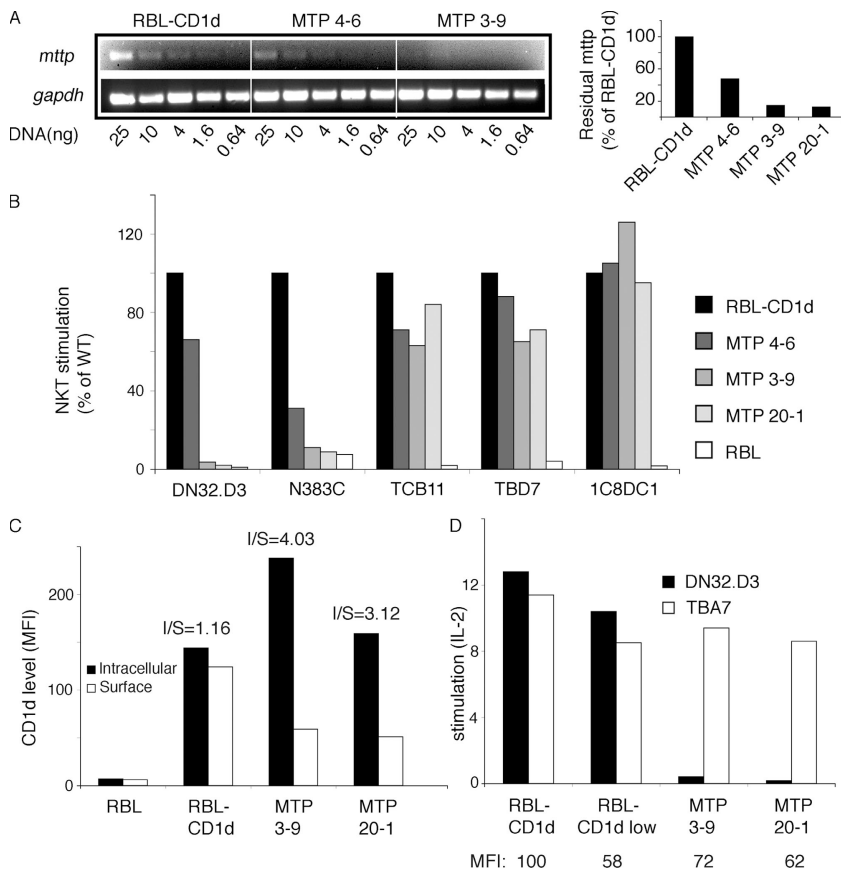

Figure 3. MTP-deficient RBL-CD1d cells fail to stimulate Vo14J $\boldsymbol{\alpha} 18$ NKT cells. (A) Various siRNA were stably expressed to generate MTP-deficient clones in RBL-CD1d cells. (left) Semiquantitative RT-PCR levels of mttp and gapdh; (right) bar graph display of residual mttp mRNA in individual MTP KD clones. (B) Stimulation of $\mathrm{V} \alpha 14-\mathrm{J} \alpha 18$ NKT hybridomas (DN32.D3 and N383C) and non-V $\alpha 14-J \alpha 18$ NKT hybridomas (TCB11, TBA7, and 1C8DC1) by individual siRNA MTP KD clones. Clone MTP 4-6 expressed $50 \%$ of the original mRNA levels of $m t t p$, whereas clones 3-9 and 20-1 expressed $<20 \%$. RBL cells expressing neither CD1d (RBL) nor siRNA (RBL-CD1d) were used as controls. Data are representative of three independent experiments. (C) Surface and intracellular levels of CD1d were detected by flow cytometry using FITC- and PE-conjugated CD1d antibodies before and after permeabilization, respectively. The ratio between intracellular and surface CD1d (I/S) is shown for each clone. (D) Stimulation of $\mathrm{V} \alpha 14-\mathrm{J} \alpha 18$ NKT hybridoma (DN32.D3) and non- $\mathrm{V} \alpha 14-$ $J \alpha 18$ NKT hybridoma (TCB11) by RBL-CD1d cells, sorted RBL-CD1d cells expressing low levels of CD1d, and by siRNA MTP KD clones. Mean fluorescence intensity of $C D 1 d$ surface levels are indicated below the graph.

NKT cells. These two compounds were very poorly presented by the MTP KD clones, even at the highest concentrations. In addition, we found a profound defect in the presentation of exogenously added iGb3 or its precursor iGb4, both of which are also highly dependent on lysosomal trafficking of CD1d.

\section{Conserved rate of CD1d biosynthesis and Golgi maturation}

Because it is a direct prediction of the "ER chaperone" model that CD1d biosynthesis should be defective in the absence of MTP, we measured the rate of CD1d biosynthesis by immunoprecipitating CD1d in $\left[\mathrm{S}^{35}\right]$ cystein and methionine metabolic pulse-chase experiments. As shown in Fig. 5 A, there was no significant decrease in the rate of biosynthesis or the rate of acquisition of Endo-H resistance, indicating intact ER synthesis and Golgi maturation of CD1d in the absence of MTP. 


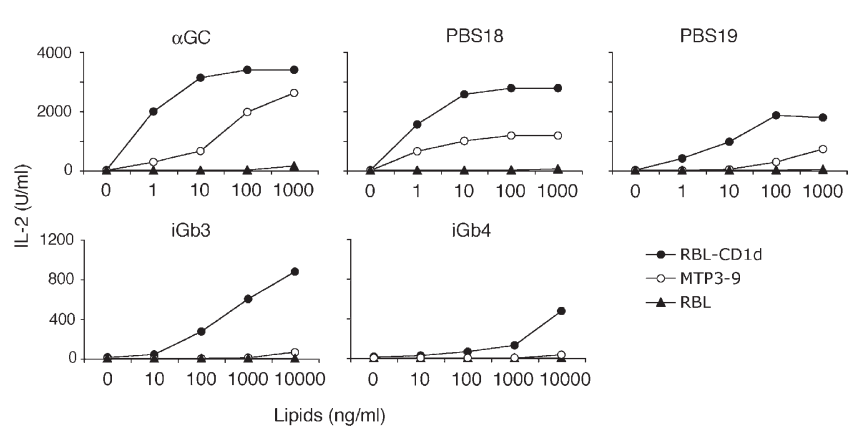

Figure 4. Defective lipid antigen presentation by MTP-deficient RBL-CD1d cells. The $V_{\alpha} 14-J \alpha 18$ NKT cell hybridoma (DN32.D3) was stimulated by various lipids, as indicated, in the presence of RBL-CD1d cells, MTP KD clone 3-9, and cells that do not express CD1d (RBL). PBS18: Gal $\alpha 1,2 \alpha$ GalCer; PBS19: Gal $\alpha$ 1,4 $\alpha$ GalCer; iGb3: Gal $\alpha$ 1,3 Gal $\beta$ 1,4 Glc $\beta$ 1,1 Cer; iGb4: GalNAc $\beta$ 1,3 Gal $\alpha$ 1,3 Gal $\beta$ 1,4 Glc $\beta$ 1,1 Cer.

\section{Defective recycling of $\mathrm{CD} 1 \mathrm{~d}$ from lysosome to plasma membrane}

Altogether, our data suggested preservation of the secretory pathway and pointed to the lysosomal compartment as the site of impact of MTP deficiency. Using surface and intracellular flow cytometry staining for CD1d in MTP mutant and wild-type cells, we observed a conspicuous and consistent three- to fourfold increase of the intracellular over surface ratio of CD1d in MTP-deficient clones (Fig. 3 C). CD1d accesses the plasma membrane at two different stages of its life cycle: early, after biosynthesis by export from the ER via the Golgi to the cell surface, and late, after internalization from the plasma membrane to endosomal compartments from which CD1d recycles back and forth to the plasma membrane. Thus, the association of conserved synthesis and accumulation in late endosomal/lysosomal compartments (see below) suggested alterations in the late internalization/recycling events between plasma membrane and lysosome. Direct measurements of internalization rates using a surface biotinylation assay with cleavable biotin (15) unambiguously established that the rate of internalization was preserved in MTP-deficient cells (Fig. 5 B, left). Another assay based on surface staining with biotinylated anti-CD1d antibodies yielded identical results (Fig. $5 \mathrm{~B}$, right). Altogether, the data pointed to a defect in the recycling of CD1d from internal late endosomal/lysosomal stores to the plasma membrane. We thus extended the surface biotinylation assay to follow the surface reexpression of a cohort of CD1d molecules that were previously internalized over a prolonged $7-\mathrm{h}$ period to ensure trafficking to the lysosome. Cleavage of surface biotin was followed by a 4-h period of incubation, and a second cleavage removed the biotin associated with surface reexpression of CD1d. In the WT clone, the amount of intracellular biotinylated CD1d was reduced by $>50 \%$ between strips I and II, demonstrating active recycling to the surface. In sharp contrast, the MTP KD clone 3-9 did not show recycling from intracellular compartments to the surface, as demonstrated by the lack of reduction of intracellular CD1d-biotin

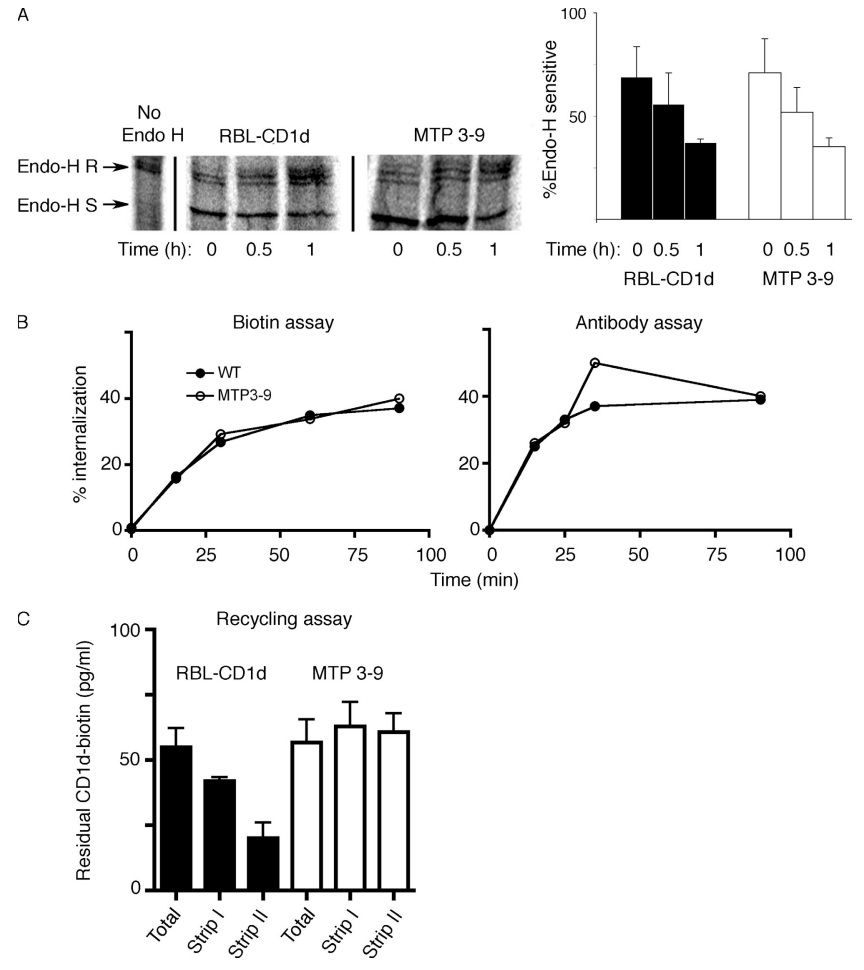

Figure 5. Biochemical studies of CD1d trafficking. (A) [ $\left.{ }^{35}\right]$ methionine and cysteine pulse-chase analysis of CD1d biosynthesis. (left) CD1d molecules were immunoprecipitated with the $2 \mathrm{OH} 2$ antibody, and samples were treated or not with Endo-H, before SDS-PAGE and detection with a phosphorimager. Data are representative of three independent experiments. (right) Data pooled from two kinetic experiments are shown as percentage Endo-H sensitive (100X sensitive/[resistant + sensitive]). (B) CD1d internalization. Data are representative of three independent experiments. (C) Recycling of CD1d. Residual intracellular biotinylated CD1d after first stripping (internalization) and second stripping (recycling). See Materials and methods. Mean + SD of triplicate samples is shown; data are representative of three independent experiments.

between strips I and II (Fig. 5 C). In fact, even the $\sim 30 \%$ reduction normally seen after strip I could not be observed, likely reflecting the progressive lysosomal accumulation over the initial 7-h period. Similar results were found with another MTP KD clone, 20-1 (unpublished data). Impaired CD1d recycling from lysosomes to the cell surface is sufficient to explain the selective presentation defect for lipid antigens that require CD1d trafficking to the lysosomes as well as the decreased surface expression of CD1d.

\section{Other lysosomal functions}

Lysosomal dysfunction has not been previously observed in MTP-deficient cells. Confocal analysis showed normal gross morphology of the LAMP1+ late endosomal/lysosomal compartment of MTP-deficient cells and normal localization of the majority of intracellular CD1d in this compartment (Fig. 6 A, left). However, because some LAMP-1+ vesicles appeared to be larger in MTP-deficient than in WT cells, we performed a FACS quantification of the Lysotracker+ 
compartment and detected a 1.7- to twofold increase in the staining intensity of MTP-deficient cells in two separate experiments (Fig. $6 \mathrm{~A}$, right). In a previous report, chemical inhibition of MTP did not impair presentation of ovalbumin to MHC class II-restricted peptide-specific OTII CD4 T cells. We directly confirmed that lysosomal degradation of hen egg lyzozyme (HEL) and horse radish peroxidase (HRP) proceeded with normal kinetics in the absence of MTP (Fig. 6 B). In addition, we studied the trafficking of exogenously administered PBS10, a fluorescent version of $\alpha \mathrm{GC}$ tagged with prodan on C6" (16), and demonstrated normal trafficking to the late endosome and the lysosome (Fig. 6 C, left). However, another fluorescently labeled lipid, BODIPY-LacCer,

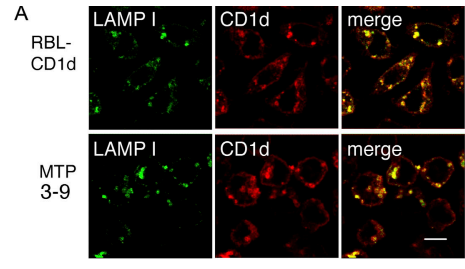

B

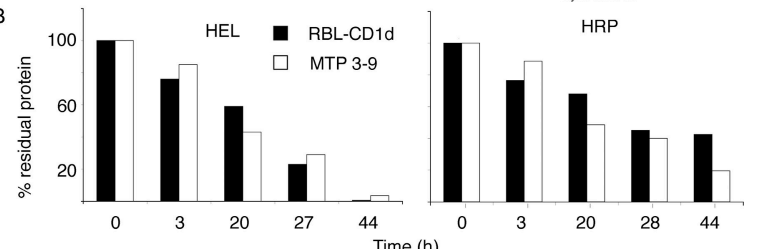

C

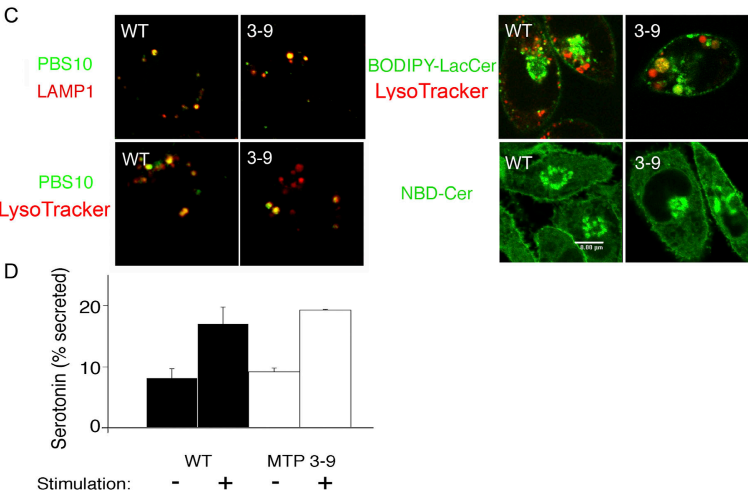

Figure 6. Lysosomal functions of MTP KD clones. ( $A$, left) Confocal microscopy analysis of RBL-CD1d and MTP KD 3-9 cells costained with LAMP1 (green) and CD1d (red). Bar, $18 \mu \mathrm{m}$. (right) Lysotracker staining fluorescence intensity of RBL-CD1d and its MTP KD variant MTP 3-9. Control indicates unstained RBL cells. The mean fluorescence intensity of MTP 3-9 was increased 1.7- to twofold over WT in two separate experiments. (B) Kinetics of HEL (left) and HRP (right) degradation, measured by Western blot or intracellular flow cytometry, respectively. Data are representative of two independent experiments. (C) Intracellular trafficking of exogenously administered lipids. (left, top) PBS10 (green) trafficking to the LAMP1 + compartment (red); (bottom) colocalization of PBS10 (green) and Lysotracker (red). (right, top) Lysosomal retention of BODIPY-LacCer in MTP 3-9 cells, shown by partial colocalization with Lysotracker; (bottom) conserved trafficking of NBD-Ceramide to the Golgi apparatus in MTP 3-9 cells. Bar, $8 \mu \mathrm{m}$. (D) $\left[{ }^{3} \mathrm{H}\right]$ serotonin release by Fc receptoractivated RBL-CD1d and MTP KD 3-9 cells. Mean + SD of triplicates wells is shown; data are representative of two independent experiments. which is known to accumulate in the Golgi after vesicular transport from the plasma membrane to the late endosome to the ER, showed abnormal retention in the Lysotracker+ compartment (Fig. $6 \mathrm{C}$, top right), suggesting the existence of at least some lipid trafficking defects in MTP-deficient cells. NBD-ceramide (Fig. $6 \mathrm{C}$, bottom right), which is rapidly transported from the plasma membrane to the ER by a nonendocytic pathway and then transferred to the Golgi by the cytosolic transporter CERT (ceramide transfer protein; reference 17), reached its destination normally in the absence of MTP. In an attempt to detect additional defects in lysosome-to-surface trafficking, we studied serotonin secretion, which occurs by granule exocytosis after exposure of RBL mast cells to IgE-DNP immune complexes (18). Fig. 6 D shows that $\mathrm{FC}$ receptor-triggered serotonin secretion was conserved in MTP-deficient RBL cells. Altogether, these results suggest the existence of partial and selective lysosomal defects associated with MTP deficiency, including an increase in the overall size of the lysosomal compartment and an abnormal lysosomal retention of the lipid BODIPY-LacCer.

\section{DISCUSSION}

Although several proteins involved in the uptake, exchange, or transmembrane transport of lipids have been clearly involved in various aspects of CD1-mediated antigen presentation (3-8), the recently reported role of MTP remains somewhat elusive. The ER location of MTP, its coprecipitation with CD1d, and its well-established function in loading apoB with lipids have suggested that MTP might be a chaperone assisting the folding of nascent CD1d proteins through lipid loading. Indeed, chaperone-assisted folding and peptide loading are essential for MHC molecules, and it is logical to postulate that similar mechanisms might be associated with CD1 biosynthesis. However, some of the reported functional consequences of the genetic ablation or chemical inhibition of MTP were not fully consistent with this proposed function. For example, the decrease in CD1d surface expression was often modest and could not alone explain the profound defects in lipid antigen presentation seen upon genetic ablation of mttp (14). Likewise, chemical inhibition of MTP in two different human cell lines led to a defect in NKT cell stimulation without notable decrease in surface CD1d levels (13). In addition, when localization of CD1d was studied by confocal microscopy of liver sections, a majority of CD1d molecules were outside the ER, whether or not MTP was inactivated (14). Finally, although purified MTP could transfer phosphatidylethanolamine onto plastic wells coated with CD1d, the lipid transfer assay was indirect, and the stoichiometry of CD1d loading was not determined (13). Whether, like the well-studied saposins (3, 4), MTP can efficiently transfer lipids onto CD1d remains unclear.

We present direct cell biological and functional evidence suggesting an alternative explanation for the CD1d-mediated lipid presentation defects of MTP-deficient cells. Metabolic pulse-chase radiolabeling experiments established that the rates of CD1d biosynthesis and transit through ER and Golgi 
were unaltered in the absence of MTP. In addition, using a panel of well-characterized NKT hybridomas reactive to endogenous lipids, we found that, in the absence of MTP, CD1d-expressing cells stimulated the hybridomas responding to endogenous lipids loaded in the secretory compartment, whereas, in sharp contrast, they failed to stimulate those recognizing lysosomal antigens such as iGb3. Consistent with these in vitro findings, NKT cell development and stimulation by thymocytes were partially impaired in vivo in chimeric mice carrying a deletion of the first exon of MTP in their cortical thymocytes.

These surprising findings led us to consider the lysosomal stage of CD1d trafficking as a potential target of MTP. Previous studies have shown that, at steady state, most of the CD1d molecules normally reside in the lysosomal compartment, from which they undergo prolonged recycling back and forth to the plasma membrane. This movement is governed by a tyrosine motif in the intracytoplasmic tail of CD1d that binds AP-2 and AP-3 (19-21). CD1d tail-truncated mutants fail to present many lipids, including the V $\alpha 14$ NKT ligand $i G b 3$, or the di- or triglycosylated derivatives of $\alpha \mathrm{GalCer}$ that require processing by lysosomal glycosidases before recognition by V $\alpha 14$ NKT cells. Thus, the functional defects associated with the tail truncation of CD1d resemble those associated with MTP deficiency.

The intracellular distribution patterns, however, are opposite. Whereas tail-truncated CD1d exhibits a higher level of surface expression and decreased intracellular accumulation, the intracellular/surface ratio is increased in MTP-deficient cells. Importantly, the intracellular site of accumulation is the late endosome/lysosome rather than the ER. Thus, the conserved internalization rate and markedly decreased recycling identify a block in the exit pathway that normally allows CD1d molecules to return to the plasma membrane after they have loaded lipid antigens in the lysosome. This anomaly provides a potential explanation for all the cell biological and functional changes of CD1d trafficking and antigen presentation observed in MTP-deficient cells or animals. Because little is known about the molecular mechanisms controlling recycling in normal cells, there is at present no clear suggestion as to the precise molecular target of MTP. Reports that MHC class II presentation is conserved in MTP-deficient cells and our own findings that lysosomal degradation of HEL and HRP proteins are unaltered, that PBS10, a fluorescently labeled $\alpha \mathrm{GC}$, traffics normally to late endosome and lysosome, and that granule exocytosis is conserved in the absence of MTP suggest that the lysosomal defects are relatively selective. However, the size of the Lysotracker+ compartment appeared to be significantly increased, and a lysosomal retention of BODIPY-LacCer, which normally reaches the Golgi after endocytosis to the late endosome, could be detected as well. Thus, changes in the lipid composition of some membranes or lipid storage may alter the dynamics of the CD1d recycling pathway. Alternatively, MTP deficiency may impact another unidentified protein involved in recycling. Notably, recent experiments investigating CD1d-mediated lipid presentation defects associated with herpes simplex virus- 1 infection have uncovered a very similar dysregulation of CD1d recycling from lysosome to plasma membrane (22), revealing the importance of this previously overlooked stage of CD1d trafficking and suggesting its relevance in host defense.

Our results do not rule out the possibility that, in the absence of MTP, aberrant lipid loading in the ER might prevent subsequent exchange with other lipid ligands in the lysosome and somehow alter CD1d recycling. This hypothesis, however, remains to be tested. Furthermore, although we found that $3 / 3$ non-V $\alpha 14$ autoreactive hybridomas (previously shown to respond to endogenous ligand loaded independently of lysosomal trafficking of CD1d) recognized CD1d in the absence of MTP, the response of another nonV $\alpha 14$ hybridoma, 14S6, was reported to be altered (13), suggesting that, in addition to the major lysosomal defects, lipid presentation in the secretory pathway might be partially impaired as well.

In conclusion, MTP deficiency induces severe defects of lipid antigen presentation by CD1d. Despite the ER location of MTP, convergent cell biological and functional experiments indicate that a major impact on CD1d-mediated antigen presentation is in the altered recycling of CD1d from the lysosome to the plasma membrane. This surprising finding reveals a previously ignored, long-range effect of MTP in an important, yet poorly understood, intracellular trafficking process.

\section{MATERIALS AND METHODS}

Mice. Mice with a "floxed" Mttp allele on a mixed C57BL/6 and 129S4/ SvJae background (B6;129S-Mttp $\left.{ }^{t m 2 S g y} / \mathrm{J}\right)$ were from The Jackson laboratory. These mice were bred with mice transgenic for $\mathrm{Mx} 1$ or Lck promoterdriven Cre recombinase on a C57BL/6 background (The Jackson Laboratory). Littermates were typed to determine Cre and floxed $m$ ttp presence by PCR according to a published protocol (23). The primer sequences used for $m t t p$ typing were 5'-GCTCTCAAGAGGAGGTTAAGG-3' and 5'-CGTCTTTCAAGAGAATGCCC-3'. Detection of Mx1 or Lck Cre by PCR was done as described (The Jackson Laboratory). MTP-deficient mice and their WT littermates (nonfloxed mttp mice with or without Cre) were used for comparative analysis. CD1 $\mathrm{KO}$ mice in the C57BL/6 background were generated and maintained as described previously (24). All mice were raised in a specific pathogen-free environment at the University of Chicago, according to the Institutional Animal Care and Use Committee guidelines.

Bone marrow radiation chimeras. C57BL/6 CD1 $\mathrm{KO}$ mice received whole-body $\gamma$-irradiation (1,000 rad) with a cesium source (Gammacell 40; MDS Nordion) and were reconstituted $6 \mathrm{~h}$ later with one i.v. injection of $10 \times 10^{6}$ bone marrow cells from various adult donors as described. Mttp $p^{\mathrm{f} / \mathrm{H}}$ Mx1-Cre mice and WT littermates were treated intraperitoneally with $400 \mu \mathrm{g}$ dsRNA (polyI:C; Sigma-Aldrich) in PBS every day for $6 \mathrm{~d}$, before bone marrow was collected and injected into irradiated CD1 KO mice.

Cell culture. Fresh thymocytes and splenocytes were obtained as described previously (25). Stably CD1d transfected RBL cells (25) were transfected with the vector pSUPER to form stable MTP KD siRNA clones (26), using selected sequences from the Rat mttp gene. The sequences used for the siRNA constructs were 5'-CCTCTGGAACCACCAATGA-3' for clone 4-6, 5'-TTGCAGCCACTCCAGATGA-3' for clone 20-1, and 5'-CTTGGAGGCTCTACGGAGA-3' for clone 3-9. Clones were selected with $12.5 \mu \mathrm{g} / \mathrm{ml}$ puromycine, and MTP mRNA levels were detected by RT-PCR (CLONTECH Laboratories, Inc.). The sense and antisense 
primers for Rat $m t t p$ were $5^{\prime}$-GTCACGATAACGGCTGTCAATGTC-3' and 5'-CCTCTCAATTTTGCATGTATCCAG-3', respectively. For rat gapdh, primers were $5^{\prime}$-CTCTGGAAAGCTGTGGCGTGATGG-3' and 5'-CTGTAGCCATATTCATTGTCATACC-3'.

Pulse-chase labeling. Experiments were done as described previously (15). In brief, to study the biosynthesis and trafficking rate of CD1d from ER to the Golgi, cells were pulsed with $0.5 \mathrm{mCi} / \mathrm{ml}\left[\mathrm{S}^{35}\right]$ methionine and cystine (GE Healthcare) and chased with excess cold methionine and cysteine at $37^{\circ} \mathrm{C}$ for the indicated times. Cells were harvested, and CD1d molecules were immunoprecipitated with $20 \mathrm{H} 2$ antibody. Samples were treated or untreated with Endo-H according to the manufacturer's instructions (New England Biolabs, Inc.), before running on SDS-PAGE gel and analysis by phosphorimager.

Biotin internalization and recycling assay. Assays were as described previously (15). In brief, for the biotin internalization assay, surface proteins were biotinylated with a cleavable biotin reagent, Sulfo-NHS-S-S-Biotin (Pierce Chemical Co.; $0.5 \mathrm{mg} / \mathrm{ml}$ in HBSS at $4^{\circ} \mathrm{C}$ for $15 \mathrm{~min}$ ). Cells were then incubated at $37^{\circ} \mathrm{C}$ for the indicated times, before stripping surface biotin with a glutathione solution. Levels of internalized CD1d molecules were detected by ELISA using 20H2 antibody to capture CD1d molecules and HRP-conjugated streptavidin (R\&D Systems) to detect biotin and quantified by reference to standard CD1d-biotin. Residual biotinylated CD1d was compared with total biotinylated CD1d at time 0 (before internalization). For the antibody internalization assay, cells were surface stained with biotinylated anti-CD1d antibody (clone 1B1; BD Biosciences), washed, and incubated at $37^{\circ} \mathrm{C}$ for the indicated times. Remaining surface CD1d molecules were detected by flow cytometry using PE-conjugated streptavidin. For the recycling assay, surface proteins were biotinylated at $4^{\circ} \mathrm{C}$ and cells were incubated for $7 \mathrm{~h}$ at $37^{\circ} \mathrm{C}$. Residual surface biotin was stripped as described above and cells were incubated for $4 \mathrm{~h}$ to allow recycling of internalized CD1d-biotin molecules to the surface. Surface biotin was stripped again, and residual biotinylated CD1d molecules were measured by ELISA as described.

Flow cytometry. CD1d- $\alpha$ GalCer tetramers were generated and used as described previously (27). FITC-conjugated anti-CD4, anti-CD1d, and anti-CD24; allophycocyanin-conjugated anti-B220; cychrome-conjugated anti-CD8; and PE-conjugated anti-CD1d were obtained from BD Biosciences. For the detection of surface and intracellular CD1d levels, RBL cells were stained with saturating amounts of FITC-conjugated anti-CD1d before wash, fixation, and permeabilization (Cytofix/Cytoperm; BD Biosciences) and staining with PE-conjugated anti-CD1d. Lysosomal staining was performed by incubating cells with LysotrackerRed DND99 (Invitrogen) at $1 \mu \mathrm{g} / \mathrm{ml}$ for $15 \mathrm{~min}$. Flow cytometry was performed with FACSCalibur (Becton Dickinson), and data were analyzed using Cell Quest pro software (Becton Dickinson).

Protein degradation assay. $1 \mathrm{mg} / \mathrm{ml} \mathrm{HEL}$ and HRP (Sigma-Aldrich) were incubated with RBL-CD1d and the MTP KD clone 3-9 for $4 \mathrm{~h}$, before the cells were washed and harvested at the indicated time points. Residual protein levels were measured by Western blot for HEL (rabbit anti-lysozyme; ab391 [Abcam]) or intracellular flow cytometry for HRP (mouse anti-HRP; ab8326 [Abcam]). Data are representative of two independent experiments.

T cell hybridoma stimulation assay. NKT hybridomas, including the V $\alpha 14-J \alpha 18$ DN32.D3 (25) and N383C (28) and the non-V $\alpha 14-J \alpha 18$ TCB11,TBA7, and $1 \mathrm{C} 8 \mathrm{DC} 1$ clones (25), were incubated at $5 \times 10^{4}$ cells per 96 flat microwell in the presence of $5 \times 10^{5}$ fresh thymocytes or $5 \times 10^{4}$ RBL cells for $24 \mathrm{~h}$ in a $0.1-\mathrm{ml}$ final volume of 1:1 mixture of Click's medium and RPMI supplemented with $10 \%$ heat-inactivated FCS, glutamine, antibiotics, and $5 \times 10^{-5} \mathrm{M} 2-\mathrm{ME}$ as described. IL-2 released in cultured supernatants was measured using CTLL-2 indicator cells as described previously (29). $\left[{ }^{3} \mathrm{H}\right]$ serotonin release assay. Determination of $\left[{ }^{3} \mathrm{H}\right]$ serotonin release was as described previously (18). In brief, $2 \times 10^{5} \mathrm{RBL}$ cells were incubated with $0.4 \mu \mathrm{g} / \mathrm{ml}$ anti-DNP IgE (Sigma-Aldrich) in the presence of $2 \mu \mathrm{Ci} / \mathrm{ml}$ $\left[{ }^{3} \mathrm{H}\right]$ serotonin (PerkinElmer) overnight. Cells were washed in modified Tyrode's buffer $\left(135 \mathrm{mM} \mathrm{NaCl}, 5 \mathrm{mM} \mathrm{Kcl}, 1 \mathrm{mM} \mathrm{MgCl}, 1.8 \mathrm{mM} \mathrm{CaCl}_{2}\right.$, $5.6 \mathrm{mM}$ dextrose, $10 \mathrm{mM}$ Hepes, and $0.1 \% \mathrm{BSA}$ ) and activated at $37^{\circ} \mathrm{C}$ with $500 \mu \mathrm{l}$ modified Tyrode's buffer containing $50 \mathrm{ng} / \mathrm{ml}$ DNP-HSA (SigmaAldrich) for $30 \mathrm{~min}$. The reaction was stopped by placing the plate on ice and adding $500 \mu \mathrm{l}$ of ice-cold modified Tyrode's buffer. Supernatant and cells were collected separately to measure serotonin release and uptake by the cells, respectively.

Confocal microscopy. To study lipid trafficking, cells were incubated with $0.5 \mu \mathrm{M}$ BODIPY-LacCer (Invitrogen) or with $9 \mu \mathrm{M}$ prodan-conjugated $\alpha$ GalCer (PBS10; reference 16 ) at $37^{\circ} \mathrm{C}$ for $1 \mathrm{~h}$ or overnight, respectively. Cells were stained with LysoTrackerRed DND99 (Invitrogen) at $0.25 \mu \mathrm{g} / \mathrm{ml}$ during the last $15 \mathrm{~min}$ and directly analyzed by confocal microscopy (without fixation) or fixed before staining with rabbit anti-LAMP1 antibodies (ab24170; Abcam) and Cy5-conjugated donkey anti-rabbit antibodies (Jackson ImmunoResearch Laboratories). To study ceramide trafficking, cells grown on coverslips were incubated with $5 \mu \mathrm{M}$ NBD-Cer (Invitrogen) at $37^{\circ} \mathrm{C}$ for $5 \mathrm{~min}$. Coverslips were washed in PBS, and samples were immediately analyzed by confocal microscopy. FITC-conjugated rat anti-mouse LAMP1 antibodies (BD Biosciences) and biotin-conjugated anti-CD1d antibodies (BD Biosciences) were used to stain LAMP1 and CD1d in RBL cells as described previously (3). Cells were examined by confocal microscopy using a confocal microscope (TCS SP2 AOBS; Leica) with a $63 \times$ NA 1.4 oil objective lens at room temperature.

The authors thank members of the Bendelac Laboratory for advice and support and Dr. Stefan Kraft for help with the serotonin release assay.

Y. Sagiv is supported by a fellowship from the Cancer Research Institute. This work was supported by National Institutes of Health grants to A. Bendelac, P.B. Savage, and L. Teyton (P01 Al053725).

The authors have no conflicting financial interests.

Submitted: 25 July 2006

Accepted: 14 March 2007

\section{REFERENCES}

1. Brigl, M., and M.B. Brenner. 2004. CD1: antigen presentation and T cell function. Annu. Rev. Immunol. 22:817-890.

2. Park, S.H., and A. Bendelac. 2000. CD1-restricted T-cell responses and microbial infection. Nature. 406:788-792.

3. Zhou, D., C. Cantu III, Y. Sagiv, N. Schrantz, A.B. Kulkarni, X. Qi, D.J. Mahuran, C.R. Morales, G.A. Grabowski, K. Benlagha, et al. 2004. Editing of CD1d-bound lipid antigens by endosomal lipid transfer proteins. Science. 303:523-527.

4. Kang, S.J., and P. Cresswell. 2004. Saposins facilitate CD1d-restricted presentation of an exogenous lipid antigen to T cells. Nat. Immunol. 5:175-181.

5. Winau, F., V. Schwierzeck, R. Hurwitz, N. Remmel, P.A. Sieling, R.L. Modlin, S.A. Porcelli, V. Brinkmann, M. Sugita, K. Sandhoff, et al. 2004. Saposin C is required for lipid presentation by human CD1b. Nat. Immunol. 5:169-174.

6. de la Salle, H., S. Mariotti, C. Angenieux, M. Gilleron, L.F. GarciaAlles, D. Malm, T. Berg, S. Paoletti, B. Maitre, L. Mourey, et al. 2005. Assistance of microbial glycolipid antigen processing by CD1e. Science. 310:1321-1324.

7. van den Elzen, P., S. Garg, L. Leon, M. Brigl, E.A. Leadbetter, J.E. Gumperz, C.C. Dascher, T.Y. Cheng, F.M. Sacks, P.A. Illarionov, et al. 2005. Apolipoprotein-mediated pathways of lipid antigen presentation. Nature. 437:906-910.

8. Sagiv, Y., K. Hudspeth, J. Mattner, N. Schrantz, R.K. Stern, D. Zhou, P.B. Savage, L. Teyton, and A. Bendelac. 2006. Cutting edge: impaired glycosphingolipid trafficking and NKT cell development in mice lacking niemann-pick type c1 protein. J. Immunol. 177:26-30. 
9. White, D.A., A.J. Bennett, M.A. Billett, and A.M. Salter. 1998. The assembly of triacylglycerol-rich lipoproteins: an essential role for the microsomal triacylglycerol transfer protein. Br. J. Nutr. 80:219-229.

10. Sharp, D., L. Blinderman, K.A. Combs, B. Kienzle, B. Ricci, K. WagerSmith, C.M. Gil, C.W. Turck, M.E. Bouma, D.J. Rader, et al. 1993. Cloning and gene defects in microsomal triglyceride transfer protein associated with abetalipoproteinaemia. Nature. 365:65-69.

11. Wetterau, J.R., and D.B. Zilversmit. 1986. Localization of intracellular triacylglycerol and cholesteryl ester transfer activity in rat tissues. Biochim. Biophys. Acta. 875:610-617.

12. Wetterau, J.R., K.A. Combs, S.N. Spinner, and B.J. Joiner. 1990 Protein disulfide isomerase is a component of the microsomal triglyceride transfer protein complex. J. Biol. Chem. 265:9800-9807.

13. Dougan, S.K., A. Salas, P. Rava, A. Agyemang, A. Kaser, J. Morrison, A. Khurana, M. Kronenberg, C. Johnson, M. Exley, et al. 2005 Microsomal triglyceride transfer protein lipidation and control of CD1d on antigen-presenting cells. J. Exp. Med. 202:529-539.

14. Brozovic, S., T. Nagaishi, M. Yoshida, S. Betz, A. Salas, D. Chen, A. Kaser, J. Glickman, T. Kuo, A. Little, et al. 2004. CD1d function is regulated by microsomal triglyceride transfer protein. Nat. Med. 10:535-539.

15. Jayawardena-Wolf, J., K. Benlagha, Y.H. Chiu, R. Mehr, and A. Bendelac. 2001. CD1d endosomal trafficking is independently regulated by an intrinsic CD1d-encoded tyrosine motif and by the invariant chain. Immunity. 15:897-908.

16. Zhou, X.T., C. Forestier, R.D. Goff, C. Li, L. Teyton, A. Bendelac, and P.B. Savage. 2002. Synthesis and NKT cell stimulating properties of fluorophore- and biotin-appended 6"-amino-6"-deoxy-galactosylceramides. Org. Lett. 4:1267-1270.

17. Hanada, K., K. Kumagai, S. Yasuda, Y. Miura, M. Kawano, M. Fukasawa, and M. Nishijima. 2003. Molecular machinery for nonvesicular trafficking of ceramide. Nature. 426:803-809.

18. Isersky, C., J.D. Taurog, G. Poy, and H. Metzger. 1978. Triggering of cultured neoplastic mast cells by antibodies to the receptor for IgE. J. Immunol. 121:549-558.

19. Sugita, M., X. Cao, G.F. Watts, R.A. Rogers, J.S. Bonifacino, and M.B. Brenner. 2002. Failure of trafficking and antigen presentation by CD1 in AP-3-deficient cells. Immunity. 16:697-706.
20. Lawton, A.P., T.I. Prigozy, L. Brossay, B. Pei, A. Khurana, D. Martin, T. Zhu, K. Spate, M. Ozga, S. Honing, et al. 2005. The mouse CD1d cytoplasmic tail mediates CD1d trafficking and antigen presentation by adaptor protein 3-dependent and-independent mechanisms. J. Immunol. 174:3179-3186

21. Elewaut, D., A.P. Lawton, N.A. Nagarajan, E. Maverakis, A. Khurana, S. Honing, C.A. Benedict, E. Sercarz, O. Bakke, M. Kronenberg, and T.I. Prigozy. 2003. The adaptor protein AP-3 is required for CD1dmediated antigen presentation of glycosphingolipids and development of Vo14i NKT cells. J. Exp. Med. 198:1133-1146.

22. Yuan, W., A. Dasgupta, and P. Cresswell. 2006. Herpes simplex virus evades natural killer $\mathrm{T}$ cell recognition by suppressing CD1d recycling. Nat. Immunol. 7:835-842.

23. Raabe, M., M.M. Veniant, M.A. Sullivan, C.H. Zlot, J. Bjorkegren, L.B. Nielsen, J.S. Wong, R.L. Hamilton, and S.G. Young. 1999 Analysis of the role of microsomal triglyceride transfer protein in the liver of tissue-specific knockout mice. J. Clin. Invest. 103 1287-1298.

24. Park, S.H., D. Guy-Grand, F.A. Lemonnier, C.R. Wang, A. Bendelac, and B. Jabri. 1999. Selection and expansion of CD $8 \alpha / \alpha^{+} \mathrm{T}$ cell receptor $\alpha / \beta^{+}$intestinal intraepithelial lymphocytes in the absence of both classical major histocompatibility complex class I and nonclassical CD1 molecules. J. Exp. Med. 190:885-890.

25. Chiu, Y.H., J. Jayawardena, A. Weiss, D. Lee, S.H. Park, A. DautryVarsat, and A. Bendelac. 1999. Distinct subsets of CD1d-restricted $\mathrm{T}$ cells recognize self-antigens loaded in different cellular compartments. J. Exp. Med. 189:103-110.

26. Brummelkamp, T.R., R. Bernards, and R. Agami. 2002. A system for stable expression of short interfering RNAs in mammalian cells. Science. 296:550-553.

27. Benlagha, K., A. Weiss, A. Beavis, L. Teyton, and A. Bendelac. 2000. In vivo identification of glycolipid antigen-specific $\mathrm{T}$ cells using fluorescent CD1d tetramers. J. Exp. Med. 191:1895-1903.

28. Zhou, D., J. Mattner, C. Cantu III, N. Schrantz, N. Yin, Y. Gao, Y. Sagiv, K. Hudspeth, Y.P. Wu, T. Yamashita, et al. 2004. Lysosomal glycosphingolipid recognition by NKT cells. Science. 306:1786-1789.

29. Bendelac, A., O. Lantz, M.E. Quimby, J.W. Yewdell, J.R. Bennink, and R.R. Brutkiewicz. 1995. CD1 recognition by mouse NK1+ T lymphocytes. Science. 268:863-865. 